\title{
Avaliação clínica de um programa computadorizado para adaptação de lentes de contato
}

\author{
Clinical evaluation of a computerized topography soft ware method for fitting contactlenses
}

\author{
Helaine Vinche Zampar ${ }^{1}$ \\ Paula Renata Caluff Lobato ${ }^{2}$ \\ Isaac Neustein ${ }^{3}$ \\ Renato Leça ${ }^{4}$
}

\footnotetext{
${ }^{1}$ Residente do segundo ano de oftalmologia do Hospital do Servidor Público Estadual, São Paulo - SP.

${ }^{2}$ Residente do primeiro ano de oftalmologia do Hospital do Servidor Público Estadual, São Paulo - SP

Chefe do Departamento de oftalmologia do Hospital do Servidor Público Estadual, São Paulo - SP

${ }^{4}$ Chefe do Setor de Lentes de Contato e Refração do Hospital do Servidor Público Estadual - SP

Endereço para correspondência: Rua Pedro de Toledo, 1800 - São Paulo (SP) CEP 04039-020.

Nota Editorial: Pela análise deste trabalho e por sua anuência sobre a divulgação desta nota, agradecemos à Dra. Cleusa Coral Ghanem.

Recebido para publicação em 18.12.2000

Aceito para publicação em 20.08.2001
}

\section{RESUMO}

Objetivo: Avaliar o desempenho do software de adaptação de lentes de contato do Topógrafo EyeSys (versão 3.10) em pacientes acompanhados do setor de lentes de contato do Hospital do Servidor Público Estadual de São Paulo, sendo alguns deles com o diagnóstico de ceratocone. Métodos: Os 29 pacientes, sendo 9 deles com diagnóstico de ceratocone, tiveram suas lentes de contato adaptadas no setor de lentes de contato deste serviço baseados na ceratometria simulada e melhor padrão fluoresceinográfico. Posteriormente, os padrões curva base e poder dióptrico das lentes adaptadas na clínica foram comparados aos mesmos padrões sugeridos pelo programa de adaptação do Topógrafo Computadorizado. Resultados: Não foi encontrada diferença estatisticamente significante nos parâmetros curva base $(\mathrm{p}=0,158)$ e poder dióptrico das lentes adaptadas $(\mathrm{p}=0,013)$, tanto nos pacientes normais como nos portadores de ceratocone $(\mathrm{p}=0,463$ para curva base e $\mathrm{p}=0,842$ para poder dióptrico); entretanto nestes encontramos valores de curva base e poder da lente maiores do que no grupo dos pacientes normais. Conclusões: O programa computadorizado EyeSys (versão 3.10) de adaptação de lentes de contato pode ser utilizado como método adjuvante para adaptação de lentes rígidas gás-permeáveis, tanto em pacientes normais como nos pacientes com ceratocone, diminuindo o tempo necessário para adaptação com maior conforto para os pacientes. Entretanto a experiência clínica continua sendo a melhor escolha.

Descritores: Lentes de contato; Topografia da córnea; Ceratocone; Software

\section{INTRODUÇ̃̃̃O}

As lentes de contato rígidas gás permeáveis são importantes alternativas para correções de ametropias, especialmente astigmatismos irregulares, apresentam boa qualidade óptica e menor aderência bacteriana e proteica, reduzindo os índices de ceratite infecciosa ${ }^{(1)}$.

Programas de videoceratoscopia computadorizada estão disponíveis para sugerir parâmetros de adaptação de lentes de contato e simular padrões fluoresceinográficos. Os parâmetros sugeridos são: curva base, diâmetro da zona óptica, diâmetro da lente, bordas da lente e poder dióptrico. Estes programas podem ser utilizados para qualquer ametropia com indicação de lentes rígidas, como pacientes com ceratocone, após ceratotomia radial e após ceratoplastia penetrante ${ }^{(2)}$.

Neste trabalho, foram comparados os parâmetros de adaptação de lentes (curva base e poder dióptrico) sugeridos pelo software (versão 3.10) do Topógrafo Eye Sys com os mesmos parâmetros encontrados na adaptação clínica para utilização como adjuvante na clínica oftalmológica. 


\section{MÉTODOS}

Foram analisados retrospectivamente 29 pacientes (55 olhos) do setor de lentes de contato do Departamento de Oftalmologia do Hospital do Servidor Público Estadual, atendidos entre os meses de janeiro e abril de 2000; destes 26 $(89,6 \%)$ eram do sexo feminino e $3(10,4 \%)$ eram do sexo masculino. A faixa etária variou entre 15 e 59 anos ( \pm 2 anos). Dos 29 pacientes analisados 9 apresentavam diagnóstico de ceratocone.

Primeiramente foram analisados os prontuários destes pacientes, onde foram verificados: refração dinâmica, biomicroscopia, topografia corneana e a lente final escolhida como ideal e indicada, que teve como base a ceratometria simulada pela topografia, o padrão fluoresceinográfico e subjetivamente o conforto referido pelos pacientes. Posteriormente os padrões curva base e poder dióptrico das lentes adaptadas na clínica foram comparados aos mesmos parâmetros dados pelo programa computadorizado de adaptação do Topógrafo Eye Sys (versão 3.10).

Para uso no software foram padronizados diâmetro de íris de $12 \mathrm{~mm}$, distância vértice de $14 \mathrm{~mm}$ e material da lente Boston RXD. Os diâmetros das lentes adaptadas na clínica foram mantidos no programa de adaptação de lentes, assim como o material das lentes foi o mesmo utilizado pelo programa computadorizado. O método escolhido e padronizado no software foi a adaptação das lentes em livramento apical.

Os dados coletados foram comparados estatisticamente aplicando-se o coeficiente não paramétrico de Wilcoxon, baseado nas medianas, que se constitui na diferença entre a mínima e máxima curvaturas corneanas. Os resultados foram demonstrados através de tabelas.

\section{RESULTADOS}

Não houve diferença estatisticamente significante nos parâmetros curva base $(\mathrm{p}=0,158)$ e poder dióptrico da lente ( $\mathrm{p}=0,103$ ) entre os 20 pacientes normais quando comparados adaptação clínica e a sugerida pelo computador, assim como entre os 9 pacientes com diagnóstico de ceratocone, curva base $(p=0,463)$ e poder dióptrico $(p=0,842)$.

A tabela 1 mostra as medidas de tendência central (média e mediana) e de dispersão (desvio padrão e amplitude de varia-

$\begin{aligned} & \text { Tabela 1. Medidas de tendência central e de dispersão, comparan- } \\ & \text { do a curva base (K) encontrado na clínica com o fornecido pelo } \\ & \text { software }\end{aligned}$
$\begin{array}{lcc}\text { K cl } & \text { K comp } \\ \text { Média } & 42,58 & 42,58 \\ \text { Mediana } & 42,37 & 42,69 \\ \text { Desvio padrão } & 1,85 & 2,02 \\ \text { Mínimo } & 40,00 & 39,00 \\ \text { Máximo } & 48,00 & 48,00 \\ \text { K cl = curva base na clínica; K comp = curva base do computador }\end{array}$

ção mínima e máxima) entre os 20 pacientes normais e a tabela 2 dos 9 pacientes com ceratocone.

Como podemos notar nas tabelas 1 e 2, os valores de média e mediana são bastante semelhantes nos indivíduos estudados A média da curva base $(\mathrm{K})$ encontrada na adaptação clínica e a fornecida pelo software nos pacientes normais foi de 42,58, o K mínimo de 40,00 da clínica e de 39,00 fornecido pelo software e o K máximo de 48,00 em ambos os métodos de adaptação. Nos indivíduos com ceratocone quando comparados com os normais, a curva base $(\mathrm{K})$ apresenta-se com valores superiores. O K mínimo encontrado foi de 42,25 comparados com 40,00 nos indivíduos sem ceratocone e a média de 44,50 contra 42,37 nos indivíduos normais como já esperado para casos de altos astigmatismos.

\section{DISCUSSÃO}

Programas computadorizados de adaptação de lentes de contato rígidas gás permeáveis representam um avanço no setor de lentes de contato, já que estas lentes apresentam um importante papel na reabilitação visual de pacientes com ametropias, especialmente em astigmatismos irregulares ${ }^{(3)}$.

Tradicionalmente as lentes são adaptadas na clínica oftalmológica baseadas na ceratometria, nos padrões fluoresceinográficos e no conforto referido pelo paciente, porém isso nem sempre é suficiente para uma boa adaptação em casos de astigmatismos irregulares $^{(2)}$.

Trabalhos comparando adaptações empírica e computadorizada (TMS-1, versão 1.41) encontraram que em 50\% dos pacientes com astigmatismo de baixo a moderado grau podem ter suas lentes adaptadas com sucesso, sem qualquer modificação nos parâmetros usando programas de adaptação de lentes de contato computadorizados ${ }^{(1)}$. Nossos resultados confirmam esses achados, já que não encontramos diferença estatisticamente significativa entre os parâmetros curva base e poder dióptrico das lentes entre a adaptação clínica e a sugerida pelo software (versão 3.10) do Topógrafo Eye Sys, tanto em pacientes normais como em indivíduos com Ceratocone.

Na comparação dos programas de adaptação do Humphrey Atlas e EyeSys Pro-Fit (versão 4.0) mostraram que os parâmetros mais modificados nestes programas foram curva base e poder dióptrico das lentes, apesar disto, as alterações

\begin{tabular}{|c|c|c|}
\hline & $\mathrm{K} \mathrm{cl}$ & K comp \\
\hline Média & 42,42 & 45,79 \\
\hline Mediana & 44,50 & 45,25 \\
\hline Desvio padrão & 2,64 & 2,51 \\
\hline Mínimo & 42,25 & 42,00 \\
\hline Máximo & 50,75 & 51,00 \\
\hline
\end{tabular}


não foram estatisticamente significantes, mostrando também que estes programas computadorizados são válidos como métodos auxiliares na adaptação de lentes rígidas gás permeáveis ${ }^{(4)}$.

O programa de adaptação de lentes (CMS) em pacientes com ceratocone, com resultados satisfatórios para a seleção da lente inicial a ser testada por eles, sendo, portanto também favoráveis aos resultados obtidos neste trabalho ${ }^{(5)}$.

De acordo com trabalhos analisados, os métodos de adaptação baseados em programas computadorizados são válidos para a escolha inicial da lente a ser testada, diminuindo o tempo gasto na adaptação proporcionando com isso maior conforto para o paciente, especialmente nos casos de ceratocone, onde a adaptação é mais trabalhosa e requer maior habilidade por parte do oftalmologista. Entretanto o número de pacientes com diagnóstico de ceratocone neste estudo foi pequeno e merece outros estudos envolvendo um grupo maior. Vale ressaltar também que estes programas não substituem o teste de lentes de provas e a prática do adaptador para alcançar o sucesso desejado.

\section{ABSTRACT}

Purpose: To evaluate the performance of EyeSys Topographic system of contact lens fitting software (version 3.10) in followed-up patients of the contact lens department of the Hospital do Servidor Público Estadual, some of them with keratoconus diagnosis. Methods: The 29 patients, 9 of them with a keratoconus diagnosis, had their contact lens fitted in this specific sector of the above mentioned hospital, based on simulated keratometry and the best fluoresceinographic pattern. Afterwards, the base curve pattern and the lens power of those lenses were compared with the same suggested patterns of the computerized topography fitting program. Results: No statistically significant differences were found regarding the base curve $(\mathrm{p}=0.158)$ and lens power of fitted lenses $(\mathrm{p}=0.013)$ both in normal and keratoconus patients ( $\mathrm{p}=0.463$ for base curve and $\mathrm{p}=0.842$ for lens power). However, in the latter group, we found greater base curve and lens power values than in the normal patient group. Conclusion: The computerized fitting program EyeSys (3.10 version) of contact lenses can be used as an auxiliary method for rigid gas permeable contact lenses, both in normal and keratoconus patients, spending less time and offering a more comfortable lens fitting. However clinical experience is the best choice.

Keywords: Contact lenses; Corneal topography; Keratoconus; Software

\section{REFERÊNCIAS}

1. Szczotka LB, Capretta DM, Lass JH. Clinical evaluation of computerized topography method for fitting rigid gas permeable contact lenses. Clao J 1994;20:231-6.

2. Lopatynsk M, Cohen EJ, Leavitt KG, Laibson PR. Corneal topography for rigid gas permeable lens fitting after penetrating keratoplasty. Clao J 1993; 19:41-4

3. Szczotka LB, Runhart W. Computerized videokeratoscopy contact lens software for RGP fitting in a bilateral postkeratoplasty patient: a clinical case report. Clao J 1995;21:52-6.

4. Binoy J, Szczotka OD. Efficiency and accuracy of two computerized topography software systems for fitting RGP lenses. Clao J 2000;26:91-6.

5. Rabinowitz YS, Garbus JJ, Garbus C, Mcdonnell PJ. Contact lens selection for keratoconus using a computer-assisted videokeratoscope. Clao J 1991; 17:88-93.

\title{
XIV JORNADA DE OFTALMOLOGIA CENTRO DE ESTUDOS PROFESSOR HEITOR MARBACK \\ 15 e 16 de Março de 2002 Fiesta Convention Center - Salvador - BA
}

\author{
Informações: Interlink Consultoria \& Eventos Ltda. \\ tel.: (71) 336-5644 - fax (71) 336-5633 \\ e-mail: id@interlinkeventos.com.br \\ home page: www.interlinkeventos.com.br
}

